\title{
Circuit simulators for circuit analysis in graduate engineering courses
}

\section{Cubells-Beltrán, María-Dolores and Reig, Càndid}

Department of Electronic Engineering, University of València, Spain.

\begin{abstract}
Circuit simulators are extensively used as an aid in many courses at the graduate level in many different engineering and applied sciences programs. SPICE (Simulation Program with Integrated Circuits Emphasis) based software programs have been used for long due to their traditional market position. If we focus on circuits analysis and linear systems subjects, the features that are required from a given simulator can be found in student/limited versions of commercial EDA (Electronic Design Automation) suites or in freewarelopen source codes. In this contribution, we analyse and compare the most revelant characteristics of a representative set of the software packages that are commonly adopted in these courses, focusing on the Spanish University system. For this purpose, the analysis (transient, DC and AC) of a typical second order passive low-pass filter is approached making use of each one. Then, we give some comments and recommendations, based on our own expertise, always taking into account the particular circumstances within a given academic scenario.
\end{abstract}

Keywords: circuit simulators; circuits analysis; teching/learning; educational technology. 


\section{Introduction}

Electrical circuits and linear systems are in the curriculum of all the programs in engineering and applied sciences. A proper teaching and understanding of these topics are key in the correct development of the career ot the students (Johnson, Butcher, Ozogul, \& Reisslein, 2014). Simple circuits containing few components can be analytically approached with regular graduate mathematics (complex numbers, arrays and calculus) or experimentally studied within basic workbenches. When circuits and systems become more complexes, or as complementary supporting tool, software simulators enter into play (Gimeno et al., 2016).

Circuit simulators have been used as pedagogical aids for long (Pota, 1997). The software SPICE (Simulation Program with Integrated Circuits Emphasis) was originally developed in 1973 by Donald O. Pederson and Laurence W. Nagel at the University of California, Berkeley. Since then, it has become a globally accepted reference in this field (Prigozy, 1989), being recognized in 2011, in its $40^{\text {th }}$ anyversary, as an IEEE Milestone (Nagel, 2011).

Nowadays, different vendors provide circuit simulators embedded in electronic design software suites with a large number of improved features including differend kind of analysis (transient, frequencial, noise ...), behavioral models for nonlinear (electronic) elements, printed circuit board design and development tools, high frequency capabilities and many others. Of course, not for free. In this scenario, a good election of a circuit simulation software for electrical circuits and and linear system at the graduate courses in engineering and applied sciences programs has turned out a major issue in the definition of the curriculum.

Up to our knowledge, the use of a particular circuit simulator has been analysed in a particular topic (Hart, 1993) or in a particular university (N. A. 0. Demerdash, 1993), but no as a comparative approach among different ones used in different universities. In this paper, we make a comparison among different options that can be found, giving recommendations depending on the particular case. After defining the scope of the study in section 2, the considered method is described in section 3. Then, conclusions are provided.

\section{Scope of the study}

As before stated, we will focus on circuit analysis and linear systems subjects included in the first courses of engineering and applied sciences programs. Slightly changing from one case to another, in the most of these courses the following topics are treated:

- $\quad$ Linear components: R, C and L. 
- Transient, DC and AC regimes.

- One or Two ports networks. Quadripoles. Transfer functions.

- Magnetic coupling. Lineal transformers,

- Laplace transform and Fourier analysis (optional)

- Transmission lines (optional)

- Passive filters (optional)

- Linear applications of operational amplifiers (rare)

The topics marked as optional are common in electrical engineering programs but are often skipped in science oriented courses. Then, the considered circuit simulator should include all the above mentioned capabilities. We have limited our study to circuit simulators that are currently being used or have been used in the recent past in graduate courses of engineering programs in Spanish universities, as detailed in Tab. 1. All of them fall withing the student version or the open software schemes.

Table 1. Circuit simulators used in engineering graduate courses in Spanish universities.

\begin{tabular}{|c|c|c|c|c|c|}
\hline University & PSpice & OrCAD PSpice & LTSpice & Microcap & QUCS \\
\hline Complutense de Madrid & 口 & & & & \\
\hline Sevilla & - & & & - & \\
\hline Extemadura & - & & & & \\
\hline Politecnica de Cartagena & - & & & - & \\
\hline Illes Balears & 口 & & & & \\
\hline Pública de Navarra & घ & & घ & & \\
\hline Cantabria & - & & & & \\
\hline Miguel Hernández & & - & & & \\
\hline Alcalá & & - & & & \\
\hline Santiago de Compostela & & घ & & & \\
\hline Cádiz & & & & - & - \\
\hline Politècnica de Catalunya & - & - & & & $\mathbf{\square}$ \\
\hline Jaén & & - & & & \\
\hline Barcelona & [ & & & & \\
\hline Politécnica de Valencia & - & - & - & - & \\
\hline Murcia & & घ & & & \\
\hline Zaragoza & & & & - & \\
\hline Carlos III & & - & & & \\
\hline Las Palmas de Gran Canaria & & & - & & \\
\hline València & - & & - & & \\
\hline Castilla - La Mancha & - & घ & घ & & \\
\hline Politécnica de Madrid & & - & & & - \\
\hline UNED & & & & घ & घ \\
\hline
\end{tabular}




\subsection{PSpice}

The most of current and past circuit simulators are based on the original kernel of SPICE, developed at Berkeley in the seventies, being SPICE3F5 the last freely released version. In fact, since then, commercial versions with enhanced tools have evolved based on the original SPICE code. PSpice was the first PC oriented Spice simulator, firstly released in 1984 by MicroSim. In 1998, MicroSim was acquired by OrCAD, which itself was subsequently purchased by Cadence Design in July 1999. In this way, the last version of MicroSim PSpice (8.0) was discontinued in 1998, but a lot of institutions are still using it, assuming that no updates or new models will be available.

\subsection{OrCAD PSpice}

OrCAD PSpice took over MicroSim PSpice with its 9.1 release, including a student vesion, which become as popular as its predecessor, remaining as the reference in the academic environment. Now we are with the $16^{\text {th }}$ version.

\subsection{MicroCAP}

First version of MicroCAP (from Spectrum Software) was released in 1982 both for Apple II and IBM platforms. Since then, it has followed a compiting evolution with PSpice, up to the current $11^{\text {th }}$ version, also releasing the corresponding student versions.

\subsection{LTSpice}

LTSpice is a SPICE based simulator, schematic capture and waveform viewer, courtesy of Linear Technology (LT), also providing electronic models of their own components. With a lot of support from LT and general users, it display comparable features with PSpice or Microcap. With the purchasing of LT by Analog Devices in 2017, the free distribution of LTSpice could be questioned.

\section{4. $Q U C S$}

The Quite Universal Circuit Simulator (QUCS) is a free-software electronics circuit simulator, released under the GPL scheme. It is intented as an easier-to-use tool, when compared with OrCAD or MicroCAP suites. It is powerful, easy-to-use and with a high number of additional featres such as transmission lines analysis, Verilog/VHDL support or allowing the use of different simulation kernels.

\section{5. $g E D A$}

It was launched as a project for providing Linux users of a good EDA suite, including Gnucap as the circuit simulator. Being an excellent tool, it will be not considered in the current study. 


\subsection{Web based simulators}

Nowadays, open web-based circuit simulators can be also considered for circuit analysys (Weyten, Rombouts, \& De Maeyer, 2009). Among the most popular, we can name PartSim ("PartSim," 2018), CircuitLab ("CircuitLab," 2018) and EasyEDA ("EasyEDA," 2018) as excellent options. The main concern on web based simulators is that there is not warranty of having it available for long, what is a handicap for the programation of future courses.

Table 2. Results on typical analysis performed with the considered circuit simulators.

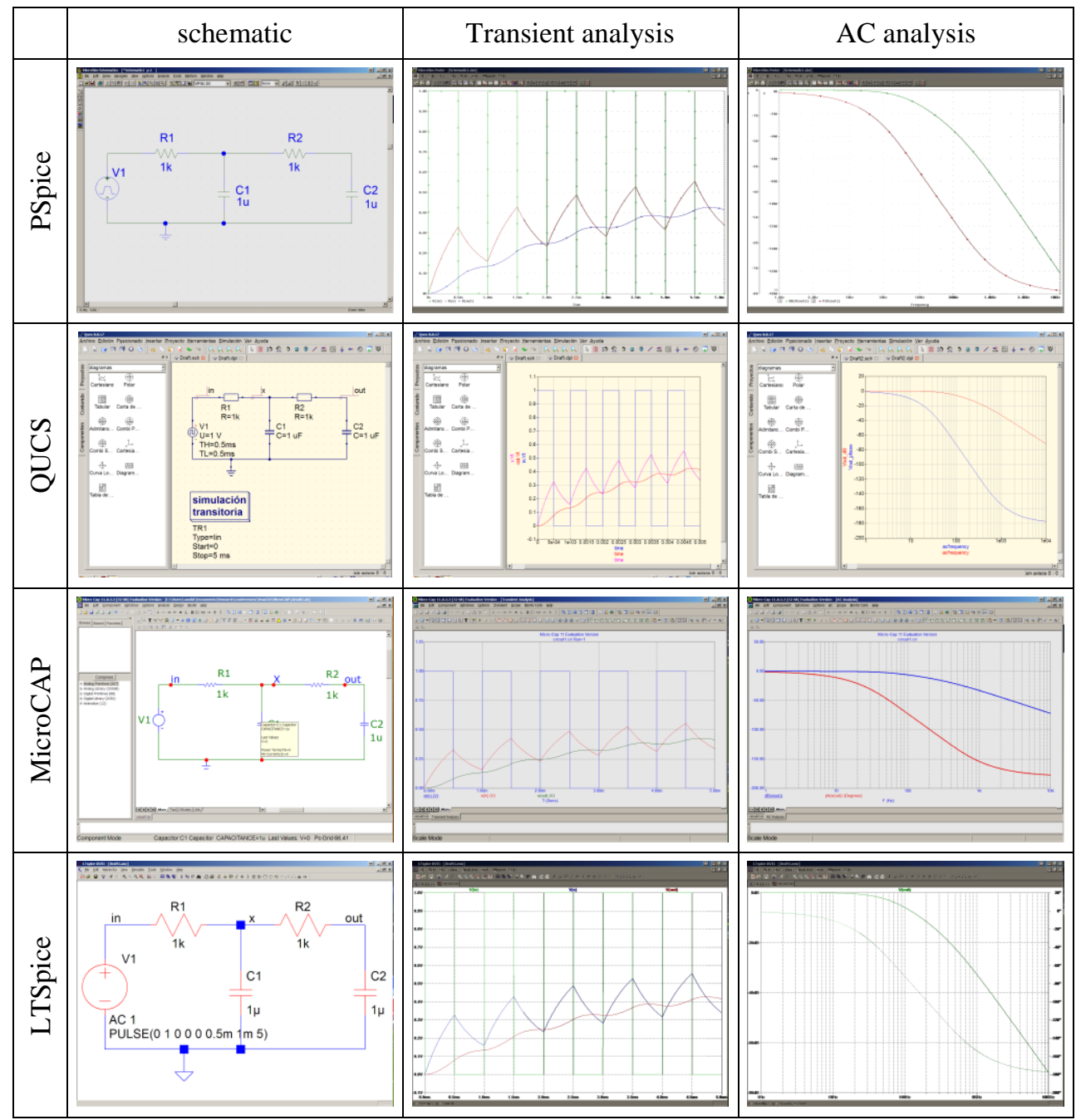




\subsection{Full versions}

Some vendors offer university programs at a reasonable price, giving access to full versions of the software. ADS from Keysight, AWR from National Instruments or Virtuoso/Systems from Cadence are valid examples of this policy. These software packages are very powerful and all of them include tools for circuit analysis. Nevertheless, they focus on specific fields of electronics (high frequeny or microelectronics) and they require advanced training efforts, not justified at first courses. In addition, the number of released licensed sites in these university programs are usually limited, which is often sufficient in postgraduate courses, but hardly compatible for scheduling different laboratory groups.

\section{Method}

In order to highlight the above exposed considerations, a representative example is approached with the different considered circuit simulators. We will consider a second order low-pass passive filter, and we will perform the typical DC, transient and AC analysis. Such kind of circuits are frequently used as testbenches for the evaluation of simulation tools and academic approaches (Coppens, Van den Bossche, \& De Cock, 2017). The results are collected in Table 2.

With proper teching guidance, freshman year students are capable to perform any of the proposed analysis in less than an hour, regardless the considered simulator. That is to say, a laboratory session dealing with the full analysis of this circuit (schematic, transient and frequency analysis) can be easily completed in three hours, which is a common duration of the laboratory sessions. If mixed experimental/simulation laboratory sessions are considered, it is a good practice to dedicate the first laboratory session as introductory both for the basic instruments and the used simulator.

In any case, all the considered circuit simulators have demonstrated its good performance in the different analysis listed at the beginning of Section 2. Parametric analysis, use of combined equations, exportation of results, compability with netlists of different origins and many other are common built-in features. In summary, all the considered simulators perfectly match the requirements for circuit analysis courses. In this regards, we should note that, in addition to the technical capabilities of the software, other characteristics play an important role in the selection process. Academically speaking, we consider as valuable the following characteristics, with valorations collected in Tab. 3:

- Freely available for the students

- Good amount of supporting documents, tutorials ...

- Good learning curve, allowing students to focus on the problem, not on the tool.

- Useful for other subjets: analog/digital electronics, VLSI design, HF circuits ... 
- Others

When possible, is highly recommendable to propose the same circuit simulator in circuit analysis matters as well as in introductory electronics courses, including digital electronics. In this way, the teaching/leaning process is optimized. In addition, the students assume the potential of the circuit simulator as a useful tool in different fields, gaining confidence in this respect. It is also considered a good practice to provide the students with laboratory scripts not linked to a particular circuit simulator. Then, a set of supporting material can be provided in parallel. In this way, the idea of the simulator as an aiding tool is better perceived by the students. In some particular cases, a certain flexibility in the use of different circuit simulators can be beneficial. The definition of the circuit by direct writing of the netlist, skipping the use of the schematic editor can also being plausible as a pedagogical exercise. Finally, depending on the specific specialization of a particular engineering degree (electrical, communications, industrial ...), the use of full versions of EDA suites can be considered in intermediate courses, as an initial training step.

Table 3. Assesment of the more relevant features of the considered circuit simulators.

\begin{tabular}{lccccc}
\hline & PSpice & $\begin{array}{c}\text { OrCAD } \\
\text { PSpice }\end{array}$ & LTSpice & Microcap & QUCS \\
\hline Full version & yes & no & yes & no & yes \\
Linux/Mac version & no / no & no / no & yes / yes & no / no & yes / yes \\
Availability & 3 & 4 & 5 & 5 & 5 \\
Schematic editor & yes & yes & yes & yes & yes \\
Learning curve & 4 & 4 & 4 & 4 & 4 \\
Graphics capabilities & 4 & 4 & 2 & 4 & 4 \\
Documentation and support & 3 & 4 & 5 & 4 & 5 \\
Export and import options & 3 & 3 & 3 & 3 & 3 \\
A/D electronics devices & $4 / 4$ & $4 / 4$ & $4 / 4$ & $4 / 4$ & $4 / 4$ \\
Additional features & PCB design & PCP design & TI models & Stability & HF analysis \\
\hline
\end{tabular}

\section{Conclusions}

The use of circuit simulators as a supporting tool in circuit analysis and linear systems courses is essential, as demonstrated for long, but it must not substitute the analytical approach and the experimental measurements. Because free versions of circuit simulators have been proved to fulfil the contents of common courses, the election of a particular one in an specific degree must be understood as an academic decision, and it must be taken after a reflection exercise, taking into account the related particular circumstances such as the nature of the degree, the background of the students or the schedule of the laboratories. The 
learning outcomes as a function of the specifically considered simulator are not easy to assess an still remain as a matter of concern.

\section{References}

CircuitLab. (2018). Retrieved from https://www.circuitlab.com

Coppens, P., Van den Bossche, J., \& De Cock, M. (2017). Student understanding of first order RC filters. American Journal of Physics, 85(12), 937-947. http://doi.org/10.1119/1.5003805

N. A. O. Demerdash, R. H. (1993). Impact of Academic Computing on Teaching Electrical Engineering at Clarkson University. IEEE Transactions on Education, 36(1), 94-102.

EasyEDA. (2018). Retrieved from https://easyeda.com

Gimeno, C., Sánchez-Azqueta, ;, Celma, C. B., Santiago, Aldea, \& Concepción. (2016). Electrónica enREDada: An experience with a webinar program. In 2nd International Conference on Higher Education Advances, HEAd'16. http://doi.org/10.4995/HEAd16.2016.2552

Hart, D. W. (1993). Circuit Simulation as an Aid in Teaching the Principles of Power Electronics. IEEE Transactions on Education, vol. 36, 10-16.

Johnson, A. M., Butcher, K. R., Ozogul, G., \& Reisslein, M. (2014). Introductory Circuit Analysis Learning From Abstract and Contextualized Circuit Representations: Effects of Diagram Labels. IEEE Transactions on Education, 57(3), 160-168. http://doi.org/10.1109/TE.2013.2284258

Nagel, L. (2011). The 40th Anniversary of SPICE: An IEEE Milestone [Guest Editorial]. IEEE Solid-State Circuits Magazine, $\quad 3(2), \quad 82$. http://doi.org/10.1109/MSSC.2011.941445

PartSim. (2018). Retrieved from http://www.partsim.com/simulator

Pota, H. R. (1997). Circuit Theory and Analog Electronics - Computer Aided Teaching. International Journal of Electrical Engineering Education, 34(2), 141-160. http://doi.org/10.1177/002072099703400205

Prigozy, S. (1989). Novel applications of SPICE in engineering education. IEEE Transactions on Education, 32(1), 35-38. http://doi.org/10.1109/13.21160

Weyten, L., Rombouts, P., \& De Maeyer, J. (2009). Web-Based Trainer for Electrical Circuit Analysis. IEEE Transactions on Education, 52(1), 185-189. http://doi.org/10.1109/TE.2008.924213 